\title{
Anglers' Riverfly Monitoring Initiative (ARMI): A UK-wide citizen science project for water quality assessment
}

\author{
Stephen J. Brooks ${ }^{1,5}$, Ben Fitch ${ }^{2,6}$, John Davy-Bowker ${ }^{3,7}$, and Soraya Alvarez Codesal ${ }^{4,8}$ \\ ${ }^{1}$ Life Sciences, Natural History Museum, London, SW7 5BD, UK \\ ${ }^{2}$ The Riverfly Partnership, c/o The Ferry Landing, Far Sawrey, Ambleside, Cumbria, LA22 OLP, UK \\ ${ }^{3}$ Freshwater Biological Association, River Laboratory, East Stoke, Wareham, Dorset, BH20 6BB, UK and Life Sciences, Natural History \\ Museum, London, SW7 5BD, UK \\ ${ }^{4}$ Freshwater Biological Association, The Ferry Landing, Far Sawrey, Ambleside, Cumbria, LA22 OLP, UK
}

\begin{abstract}
The Anglers' Riverfly Monitoring Initiative (ARMI) is a UK-wide citizen science project focused on river water quality assessment. There are currently $>2000$ ARMI volunteers monitoring $>1600$ sites that are organized into 35 regional hubs across the UK. ARMI is effective in the early detection of water pollution and complements the routine monitoring undertaken by the UK statutory environment agencies. ARMI volunteers are trained to take standardized 3-min kick-samples of freshwater invertebrates from a river site, and use these samples to produce an ARMI score based on the abundance of key pollution-sensitive taxa. ARMI scores and standard invertebrate monitoring metrics are closely correlated. Each sampling site has a 'trigger level' score set by the national regulatory authority-e.g., the Environment Agency (EA) in England. If the ARMI score falls below this trigger level, the regulatory authority is notified and agency officers investigate the cause of the low score. This process has resulted in many reports of pollution incidents that otherwise may have gone undiscovered but were instead rapidly detected and neutralized. In some cases, investigations resulted in fines being levied against those responsible. ARMI data have also proved useful in assessing the effectiveness of river restoration schemes. Here, we demonstrate the effectiveness of the ARMI as a structured citizen science program in enhancing the environmental protection of rivers. We also show that the ARMI program complements the work of statutory authorities and describe how it promotes community engagement with river environments.
\end{abstract}

Key words: Riverfly Partnership, Anglers' Riverfly Monitoring Initiative, pollution, citizen science, river quality assessment, biomonitoring, volunteer, Environment Agency, community engagement, freshwater, macroinvertebrates

There is increasing interest from national and local governments, academics, and community groups in the role that citizen science can play in augmenting and complementing statutory environmental monitoring schemes (Roy et al. 2012). The use of volunteer citizen scientists is seen as a means to save public money, increase the amount of environmental data available, improve the local environment, and help localities meet conservation goals (Bonney et al. 2009). Further, the use of volunteer citizens raises public awareness of local environmental issues, increases democ- ratization by including local communities in decision making processes, improves scientific literacy, improves public health and well-being through participation in practical outdoor work, and enhances community cohesion (Bonney et al. 2009). For these citizen science schemes to be successful they must produce useful and reliable data, and they must attract and engage participants. Thus, the attention of citizen science project organizers, ecological and social scientists, and end-users has focused on both quality assurance of outputs and motivation of volunteers.

E-mail addresses: ${ }^{5}$ s.brooks@nhm.ac.uk; ${ }^{6}$ ben@riverflies.org; ${ }^{7 j d b @ f b a . o r g . u k ; ~}{ }^{8}$ salvarez@fba.org.uk

DOI: 10.1086/703397. Received 2 June 2017; Accepted 22 August 2018; Published online 11 April 2019.

Freshwater Science. 2019. 38(2):270-280. (C) 2019 by The Society for Freshwater Science. 
The engagement of citizen scientists in monitoring river water quality has been gaining traction worldwide because of the important amenity value that local communities often place on rivers. In particular, successful projects have been reported from New Zealand (Moffett and Neale 2015, Storey et al. 2016), Australia (http://www.vic.waterwatch.org .au/cb_pages/welcome_to_waterwatch_victoria.php), USA (Overdevest et al. 2004, Gowan et al. 2007, Latimore and Steen 2014), and Canada (Reynoldson et al. 1986, Savan et al. 2003). In Britain, there is a widespread perception among citizen stakeholders, especially anglers, wildlife enthusiasts, and local community groups, that river water quality is declining (Environment Agency and English Nature 2004, WWF-UK 2014). These stakeholders are also concerned that the relevant regulatory authorities appear unable to halt or even slow this decline because of increasingly inadequate resources. When confronted with official data, which may contradict their own perceptions and suggest stable or improving river quality, we have found that citizen groups may become frustrated and feel disenfranchised from the decision-making process. However, if citizens can generate their own data on river quality, with standardized methods to produce reliable water quality assessments, then they can develop their own evidence base and begin an informed dialogue with the regulatory authorities. Citizen science can therefore result in closer working partnerships between citizens and regulatory bodies as well as improved river ecosystems. The approach outlined above is the principle behind the Riverfly Partnership (RP), which was formed in the UK in 2004 to provide a forum for river stakeholders. The Anglers' Riverfly Monitoring Initiative (ARMI) was officially launched in 2007, under the auspices of the RP, and provides a means for citizens to assess river water quality.

The RP is a network of organizations and individuals, including anglers, conservationists, scientists, water course managers, and regulatory authorities, that work together to protect and enhance the quality of British rivers and conserve riverflies (i.e., Trichoptera, Ephemeroptera, and Plecoptera) and their habitats (www.riverflies.org). The ARMI was developed by the RP, in conjunction with the Environment Agency (EA) of England, to provide a means for citizens to monitor the water quality of their local rivers. RP is not a membership organization, instead both RP and ARMI provide a conduit through which individuals and local groups can organize themselves and accumulate data on the quality of their local river environment. When citizens volunteering for the ARMI detect serious pollution incidents, they inform the regulatory authority, which then takes the appropriate action to mitigate the incidents.

The focus of this paper is to review the effectiveness of ARMI as a citizen science project that complements and augments the routine statutory river monitoring. We first describe the ARMI methods and outline its organizational structure. We next present an analysis, both visual and sta- tistical, of the comparison between citizen science ARMI index scores and regulatory authority biological quality scores across England and Wales. We then present several case studies that evaluate the effectiveness of ARMI in detecting pollution incidents and also the potential of ARMI to monitor the effectiveness of river restorations. Finally, we consider how stimulation of volunteer motivation may encourage long-term engagement of volunteers.

\section{METHODS \\ ARMI methods}

The ARMI protocol is a simplified version of the routine biomonitoring methods used by the regulatory agencies in the UK. Volunteers use a standard sampling net (250-mm frame and 500-mm deep net bag with 1-mm mesh) to sample each site by taking a 3-min kick sample from the range of habitats present at the site. The volunteer then spends 1-min selecting large stones from the river bed and handwiping them in the mouth of the net to dislodge organisms which may not have been collected in the kick sample. The volunteer subsequently cleans the net contents of fine silt, empties the remaining contents into a sorting tray, and estimates the $\log _{10}$ scale abundance of 8 macroinvertebrate target taxa. These taxa (cased Trichoptera (cased caddis), caseless Trichoptera (caseless caddis), Ephemeridae (mayfly), Ephemerellidae (blue-winged olive), Baetidae (olives), Heptageniidae (flat-bodied mayfly), Plecoptera (stonefly), and Gammaridae (shrimp)) were chosen because they are easy to identify at this taxonomic resolution, cover a range of sensitivities to pollution, have national applicability, are present year-round (with the exception of Ephemerellidae), and are familiar to most anglers. An ARMI score for the site is then generated by allocating a score of 1 to 4 according to the $\log _{10}$ abundance category of each target taxon and summing the scores of all the target groups. Local regulatory authorities give each sampling site a trigger level score based on their long-term data for the site. Higher ARMI scores indicate higher river quality, and the trigger level is set significantly below the expected ARMI score for the site. An ARMI score at or below the trigger level will therefore indicate that a serious pollution incident has occurred. If the trigger level is breached, the ARMI volunteer confirms the breach by resampling the site and then informs the local agency officer who will investigate the cause and take appropriate action.

Prior to participating in the ARMI scheme, the volunteers are trained in the ARMI protocol at a 1-d workshop. Training is conducted by an RP-accredited tutor, and the local regulatory authority officer usually attends and assists with the training. Each participant is given a laminated foldout chart that provides a simple identification guide to the 8 target invertebrate taxa, a description of the sampling protocol and scoring mechanism, and instructions on what to 
do if the trigger level is breached. The chart also includes information on how to upload results to the national ARMI database and guidelines on health, safety, and biosecurity. The training workshop is usually located at a venue close to the sites the volunteers will monitor and consists of a classroom session in the morning and a practical session on the river in the afternoon. When a volunteer has successfully completed the training workshop, they are issued a certificate.

ARMI volunteers are encouraged to monitor their sites monthly throughout the year. The ARMI scores are not expected to replicate or be a substitute for the results of the regulatory authority's routine monitoring program. Instead, they are intended to complement routine monitoring and provide an early warning of potential water quality problems (Di Fiore and Fitch 2016). In England, the EA routinely samples their biomonitoring sites $2 \times / y$ every 3 to $5 \mathrm{y}$, whereas ARMI sampling is done monthly at multiple sites on a river.

\section{ARMI structure}

Volunteers and ARMI groups self-select the river they will monitor. Typically, this river will be close to where they live or will be a river that they fish regularly. Monitoring sites are scrutinized for suitability (e.g., health and safety risks) and approved by an officer from the regulatory authority. Volunteers are encouraged to select sites that are not currently being monitored by the regulatory authority. These sites are often above and below a possible pollution source and sometimes include the defunct sites of the regulatory authority. Post-sampling, the volunteer uploads their site records (i.e., abundance of each of the 8 invertebrate target groups and the ARMI score for the site) onto the national ARMI database, which has open access. A volunteer coordinator for each river verifies the records for that river before the records go live on the national ARMI database. The river coordinator provides feedback to each of the river monitors, maintains contact with the relevant officer at the regulatory authority, follows up trigger level breaches, and identifies potential new volunteers and potential new monitoring sites. As the number of volunteers grows within a region or river catchment it becomes efficient to establish a regional ARMI hub. Each hub is typically hosted by an NGO, such as a Wildlife Trust, Rivers Trust, or an angling organization. Hub coordination is often part of the job of a paid staff member of the NGO or angling organization, though the role is sometimes fulfilled voluntarily. The hub coordinator typically organizes training workshops for new ARMI recruits, seeks to accredit new volunteer tutors, and provides feedback to ARMI volunteers through regular newsletters, refresher workshops, and occasional 1-d information meetings. The hub may have a management committee constituted of the hub co- ordinator, local ARMI river coordinators, and representatives from the local regulatory authorities. Currently, there are $>2000$ active ARMI volunteers in the UK that monitor $>1600$ sites within 35 regional hubs (Fig. 1). ARMI is coordinated nationally by a full-time project manager who oversees the national ARMI database and provides communications and feedback through the RP website, quarterly newsletters, regular articles in relevant publications, a Facebook page, and a Twitter feed. The national project manager maintains, develops, and expands the ARMI network by setting up training workshops in areas that currently have poor ARMI coverage. The project manager also expands the ARMI remit by developing, managing, and promoting various optional add-on monitoring schemes (socalled Riverfly Plus, see below). ARMI is currently hosted by the Freshwater Biological Association, but it is an independent program that is managed by an executive committee steered by the Riverfly Partnership Board.

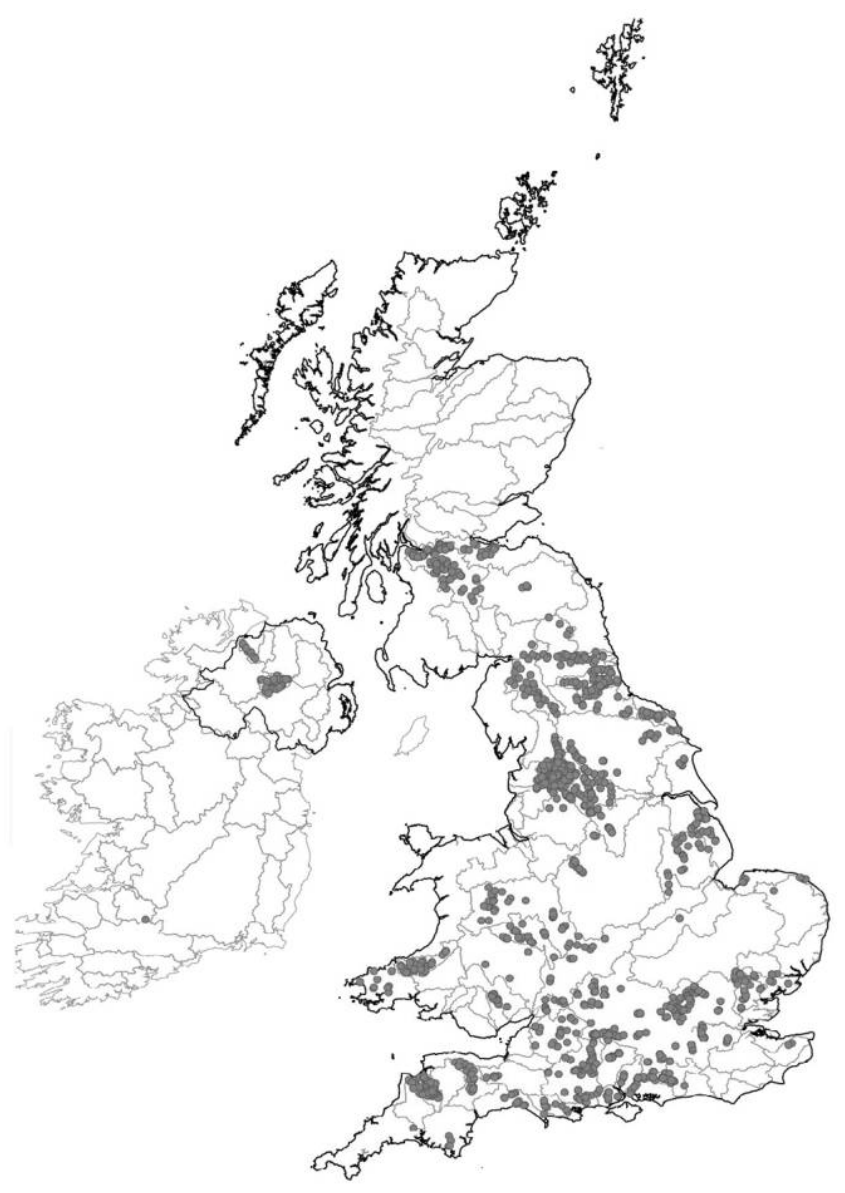

Figure 1. Location of sites monitored by the Anglers' Riverfly Monitoring Initiative (ARMI) volunteers in Britain and Ireland. The black lines denote the United Kingdom, and the gray lines are river basins. 


\section{Riverfly Plus}

As volunteers become more confident and familiar with the ARMI methods and identification of invertebrate taxa, many want to develop their skills and learn more about the river ecosystems that they are monitoring. To encourage volunteer motivation and deeper engagement in the project, the ARMI is developing several optional add-ons to be incorporated and carried out alongside the basic ARMI system. These additional monitoring activities are known as 'Riverfly Plus' and are outlined below.

Restricting the number of invertebrate target groups in the basic ARMI protocol to 8 easily recognizable taxa helps ensure that consistent data is generated even from novice volunteers. However, this taxonomic restriction means that ARMI may be sensitive only to major pollution incidents that remove a large part of the macroinvertebrate assemblage from a river. To provide a more nuanced assessment, it is necessary to add more invertebrate groups to the ARMI protocol. Adding more taxonomic groups requires that volunteers improve and expand their taxonomic identification skills. New protocols for more nuanced monitoring have been developed by Richard Chadd and Chris Extence (Environmental Agency), John Davy-Bowker (Freshwater Biological Association), and Angus Menzies (Dorset Wildlife Trust). These protocols expand the number of target taxa from 8 to 33 and include additional groups of flatworms, mollusks, annelids, crustaceans, Megaloptera, and Hemiptera. These additional taxa enable volunteers to detect the impacts of fluctuations in water quality, water flow, and siltation. By including additional taxa that occur at high frequencies in poor quality sites, this extended protocol is more suitable for poor quality urban rivers than the basic ARMI method. Importantly, these methods retain the bankside sampling technique that has been a hallmark of success with the 8-group ARMI system. The Extended Riverfly Scheme is currently being trialed in a variety of workshops nationally to assess its suitability for use across the UK.

An even more taxonomically advanced scheme is the River Invertebrate Identification and Monitoring (RIIM) index. In this scheme, trained volunteers use a 'River Invertebrate Larvae' identification application (app), which includes annotated, high-quality images, to enable the volunteer to achieve species-level identification of their samples. The dataset can then be sent to Aquascience Consultancy Ltd for biometric analysis to provide a ranking of the likely impacts on the site of excessive siltation, flow alteration, total phosphorus concentration, and organic pollution, together with an assessment of biological quality of the site (http://www.salmon-trout.org/uploads/file/RiverflyProfiling AndUsingTheApp.pdf).

Additional new protocols are also being developed and trialed to allow ARMI volunteers to monitor ecosystem function and assess the effectiveness of river restoration on ecosystem recovery (http://www.riverflies.org/ecosystem -function-and-river-restoration-monitoring). In these schemes, volunteers use cotton or paper strips, tea bags, and simple home-made colonization traps to measure invertebrate and microbial decomposition rates, invertebrate diversity and biomass, and functioning of the hyporheic zone (http:// www.riverflies.org/scratching-below-surface). Other Riverfly Plus options include the Freshwater Watch water chemistry analyses, hydromorphological surveys (MoRPh) (https:// modularriversurvey.org), monitoring for the acanthocephalan fish parasites Pomphorhynchus laevis and P. tereticollis observed as 'red spot' in Gammarus (http://www.riverflies .org/worming-your-way-pomphorhynchus-story), and recording invasive species.

\section{Data analyses}

The regulatory authorities until recently have used the longestablished Biological Monitoring Working Party (BMWP) scoring system to assess river water quality based on 82 taxa (Hawkes 1997). BMWP has now been superseded by an expanded index, the Walley Hawkes Paisley Trigg (WHPT) scoring system (Walley and Hawkes 1996, 1997, Paisley et al. 2007 ) that includes more taxa and $\log _{10}$ abundance weighting. The regulatory authorities need to be confident that the ARMI system, which is effectively a simplified version of BMWP carried out by volunteers, can provide comparable information on river water quality. To examine whether the two methods give comparable results, we used 1990 archived River Quality Survey (RQS) data collected by the National Rivers Authority (forerunner of the current Environment Agency and Natural Resources Wales). We chose data from 1990 because at that time river quality was generally poorer than it is now, so the dataset includes sites with a wider range of environmental quality than can be readily found today. The samples were analyzed in National Rivers Authority laboratories to BMWP taxonomic resolution and the 3 BMWP biomonitoring indices: BMWP score, number of taxa (NTaxa), and average score per taxon (ASPT) were calculated.

We examined the comparability of the BMWP scores and ARMI scores by mapping the indices across England and Wales so that their mutual coherence across regions with differing degrees of environmental stress could be visually assessed. We then tested whether the BMWP indices and ARMI scores gave comparable results by correlating (Pearson's $r$ ) ARMI scores with BMWP scores and ARMI scores with BMWP NTaxa across the whole of England and Wales and also within their ten constituent water management regions.

In addition to assessing the similarity of BMWP and ARMI scores geographically, we also present several case studies to highlight the utility of the ARMI system as a tool for assessing the impacts of pollution and as a method for assessing potential improvements resulting from river restoration. 


\section{RESULTS}

\section{Comparison of ARMI and BMWP indices}

The visual comparability of BMWP scores and ARMI scores across England and Wales is evident in the 1990 RQS data (Fig. 2). Both indices had higher values in upland areas in the north, west, and southwest, and lower values in the midlands and the east. Superimposed upon this natural, stream-type driven pattern are regions where pronounced environmental degradation was present, notably in the metropolitan areas of Greater Manchester, Birmingham, and northeast England. Here environmental stress resulted in lower quality rivers in 1990 and this reduced BMWP scores and ARMI scores alike.

The correlation between ARMI and BMWP scores was very strongly positive across England and Wales as a whole (Fig. 3 and Table 1). In the Anglian region, ARMI and BMWP scores were somewhat less strongly correlated ( $r=$ 0.66), but they were strongly correlated in the other 9 water management regions of England and Wales $(r=0.80-0.92)$. These correlations support the visual comparison of BMWP and ARMI scores (Fig. 2.) and indicate that ARMI scores lead to comparable inferences as BMWP scores.

Correlations between ARMI scores and BMWP NTaxa values were weaker than ARMI-BMWP correlations but still convincing (Table 1). Across England and Wales as a whole, ARMI scores and BMWP NTaxa values were highly correlated $(r=0.77)$. Correlations between sites were less strong in 5 water management regions (Anglian, Midlands, Southern, Welsh and Wessex) $(r \geq 0.6-0.78)$ but strongly so $(r \geq 0.8)$ in 5 other regions (Northumbrian, North West, South West, Thames and Yorkshire).

As judged by its correlations with the well establish BMWP score and BMWP NTaxa indices, the 8 taxonomic group ARMI score therefore appears to give broadly equivalent results, and we conclude that the ARMI system can provide a comparable approach for assessing river water quality.

\section{Detection of pollution incidents-case studies}

River Crane The River Crane runs through $35 \mathrm{~km}$ of urban conurbation in west London and has a catchment of $125 \mathrm{~km}^{2}$. Half a million people live within a short walk of the river. Since 2014, 11 sites have been monitored along the river on a monthly basis by ARMI volunteers affiliated with the Citizen Crane community project (http://www.cranevalley .org.uk/projects/citizen-crane.html). The steering group for this project includes staff of the EA, Thames Water, and Green Corridor. This steering committee links the project closely to the catchment partnership, because Green Corridor is the catchment partnership host. ARMI data shows that water quality is best in the lower parts of the River Crane, downstream of the confluence with the Upper Duke of Northumberland's River, which introduces good quality wa-
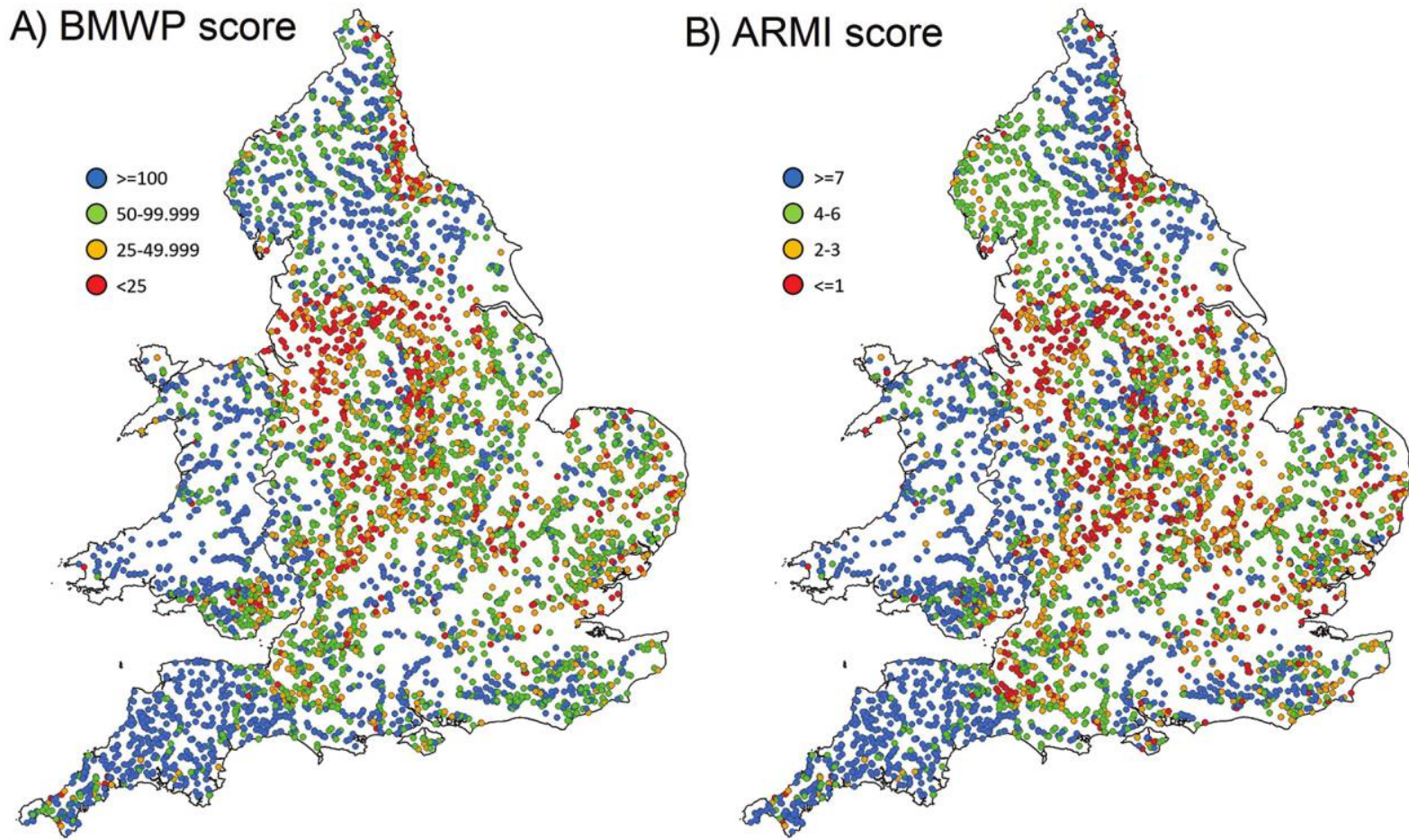

Figure 2. Biological Monitoring Working Party (BMWP) scores across England and Wales based on National Rivers Authority 1990 River Quality Survey data (A) and Anglers' Riverfly Monitoring Initiative (ARMI) scores based on National Rivers Authority 1990 River Quality Survey data (B). 


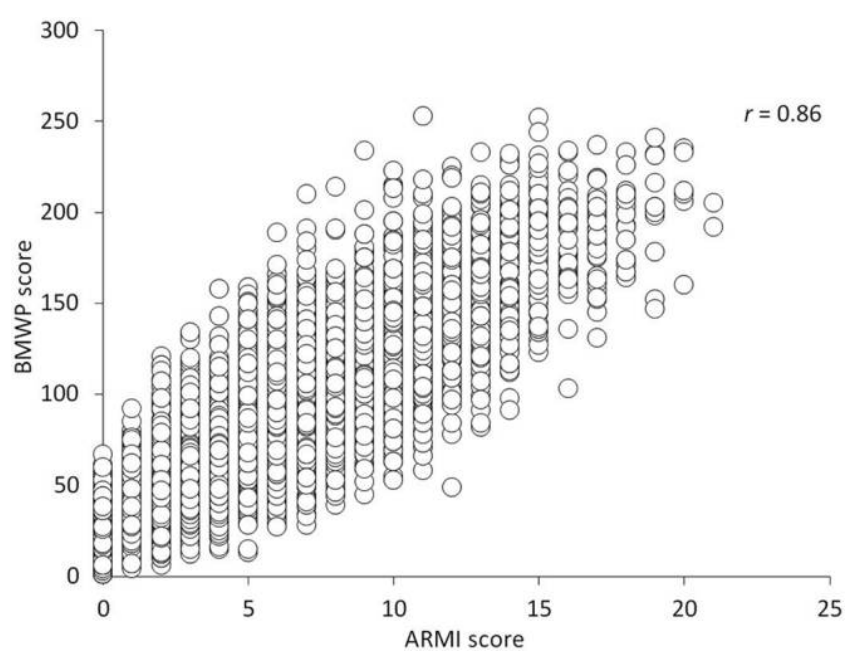

Figure 3. Relationship between Biological Monitoring Working Party (BMWP) and Anglers' Riverfly Monitoring Initiative (ARMI) scores based on 4,798 National Rivers Authority spring 1990 River Quality Survey samples.

ter. Several sites in the upper and middle stretches of the River Crane frequently breach ARMI trigger levels (J. Pecorelli, Zoological Society of London, personal communication). In $3 \mathrm{y}$ of sampling, ARMI volunteers have notified the EA of over 10 trigger level breaches that have subsequently been resolved. Water chemistry samples collected by volunteers are analyzed at the Thames Water laboratories for phosphate and ammonia. Volunteers also provide river flow assessments derived from a simple flow gauge system, which allow concentration data to be turned into pollutant loadings. These data established that the likely cause for the trigger level breaches were high concentrations of phosphates and ammonia coming from misconnections of domestic appliances via surface water outfalls, and cross-connections between foul and surface water sewerage systems. Volunteers, in conjunction with the Zoological Society of London, identified the locations of these connections through the $1^{\text {st }}$ volunteer led 'Outfall Safari' of the entire river corridor. Data from the Outfall Safari have been used to modify the 'Surface Water Outfall Program' put out by Thames Water to tackle misconnections. The methods for the Outfall Safari are now being adopted for other urban rivers across London.

River Kennet Since 2007, ARMI volunteers have been monitoring the River Kennet, one of the most famous trout fisheries in the world, in Hampshire, UK. The ARMI volunteers are coordinated by Action for the River Kennet (ARK), the local ARMI hub. The River Kennet has a catchment of $1200 \mathrm{~km}^{2}$ and an active ARMI network of 54 trained monitors that sample 49 sites (http://www.riverkennet.org /get-involved/riverfly-monitoring). A serious pollution incident that affected $15 \mathrm{~km}$ of the river was discovered by ARK's ARMI volunteers in July 2013 (Thompson et al. 2015). Monthly ARMI data were available from the site for $2 \mathrm{y}$ prior to the incident. The pollution incident caused a significant decline in all of the ARMI target taxa that were present at the site, resulting in a trigger level breach that was reported to the EA. Investigations by an EA team detected contamination by the organophosphate chlorpyrifos at $0.52-0.82 \mu \mathrm{g} / \mathrm{L}$ coming from the main tertiary sewage treatment works. Ephemeroptera abundance declined the most, whereas Trichoptera abundance declined the least. Gammarus pulex (Gammaridae) abundance also declined markedly. Within 2 months of the spill, ARMI scores had returned to pre-spill

Table 1. Pearson product-moment correlation coefficients between Anglers' Riverfly Monitoring Initiative (ARMI) and Biological Monitoring Working Party (BMWP) scores and ARMI scores and BMWP number of taxa (NTaxa) values overall and by region for samples collected during the spring 1990 River Quality Survey by the National Rivers Authority.

\begin{tabular}{lccc}
\hline & & ARMI and BMWP scores & ARMI and BMWP NTaxa \\
\cline { 3 - 4 } Region & $n$ & $r$ & 0.77 \\
\hline All regions & 4798 & 0.86 & 0.60 \\
Anglian & 796 & 0.66 & 0.84 \\
Northumbrian & 357 & 0.86 & 0.88 \\
North West & 418 & 0.91 & 0.75 \\
Midlands & 810 & 0.84 & 0.66 \\
Southern & 336 & 0.80 & 0.82 \\
South West & 478 & 0.81 & 0.80 \\
Thames & 229 & 0.81 & 0.78 \\
Welsh & 676 & 0.83 & 0.73 \\
Wessex & 361 & 0.83 & 0.89 \\
Yorkshire & 337 & 0.92 & \\
\hline
\end{tabular}


values. Ephemeroptera abundance recovered more quickly than G. pulex abundance (Fig. 4A-D).

Broughton Beck Broughton Beck meets the River Aire near Skipton, North Yorkshire, UK and is monitored monthly by volunteers from the Mitre Angling Club. The European Union's Water Framework Directive classification for invertebrates on the river is high, which is reflected in high ARMI scores. The Gatehouse Point sampling site, which is downstream of the outfall of the Broughton sewage treatment plant, usually records ARMI scores between 10 and 14, which reflects a high abundance of all 8 ARMI target taxa. In September 2015, however, the ARMI score fell to 6 (Fig. 5A), and all target groups, with the exception of Baetidae, either disappeared or declined severely. ARMI scores in November 2015 were still lower than earlier in the year but had begun to recover. Similar declines in ARMI scores were recorded again in September 2016 (Fig. 5B), which suggested a seasonal problem on the river. Both low score events were reported to the EA, which followed up with an investigation and detected a poor-quality discharge from the sewage treatment works. This observation prompted a joint in- vestigation by the EA and Yorkshire Water, which identified a problem that was previously unknown to the EA. The EA asked the sewage treatment works to improve the water quality of the outfall, and as a result a protocol is currently in place to rectify the problem. This problem would not have been detected by the EA and the high classification for invertebrates from the Water Framework Directive might have been compromised without the regular monthly monitoring by ARMI volunteers.

The River Derwent ARMI volunteers from the Tyne Rivers Trust undertake monthly sampling of the Stanley Burn, near Prudhoe, Northumberland, UK, and have detected pollution incidents several times. In November 2009 a bright yellow discharge was noticed coating the substrate of the burn. The incident was reported to the EA, who visited the site the next day and established that the discharge was coming from a building site. The EA and the developers agreed on a remediation plan and implemented it within 4 days of the incident being reported. Subsequent ARMI scores recovered to previous higher levels.
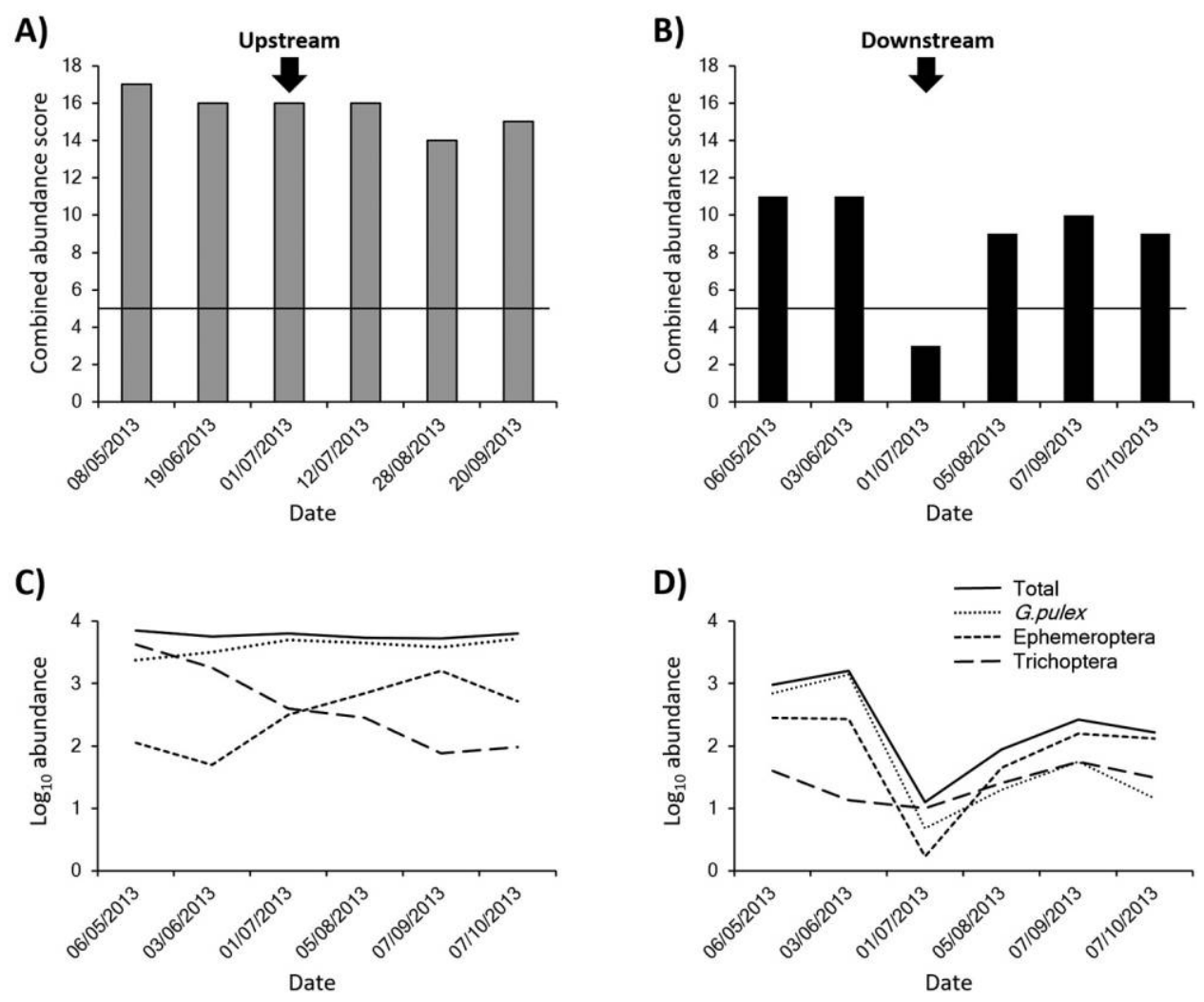

Figure 4. Anglers' Riverfly Monitoring Initiative (ARMI) scores before and after and above (A) and below (B) the toxic spill (arrows), based on total abundance of the 8 target taxa. The horizontal line represents the trigger level indicating severe environmental degradation, set by the Environment Agency. Abundance of key taxa and the total abundance of the 8 target taxa before and after and above (C) and below (D) the toxic spill. Upstream control is at Stonebridge Lane and downstream impacted site is at Elcot Mill, Marlborough, Wiltshire, UK (redrawn after Thompson et al. 2015) 
A)

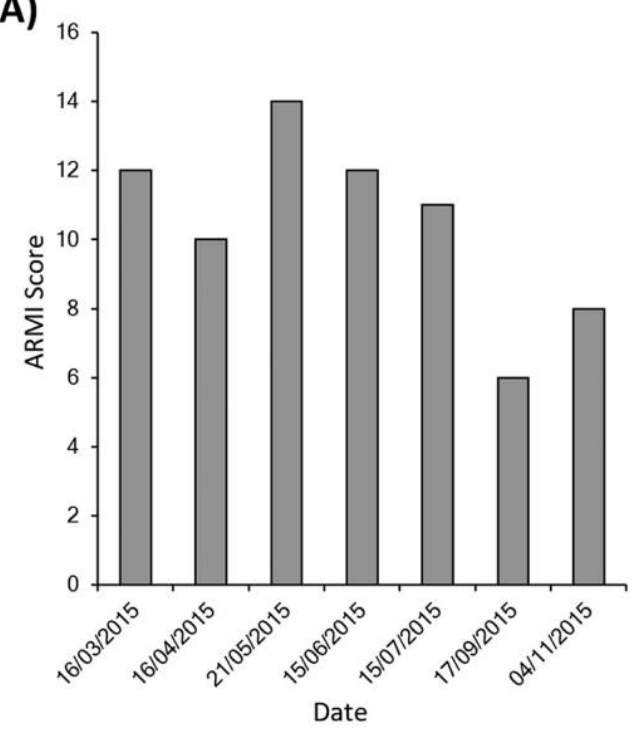

B)

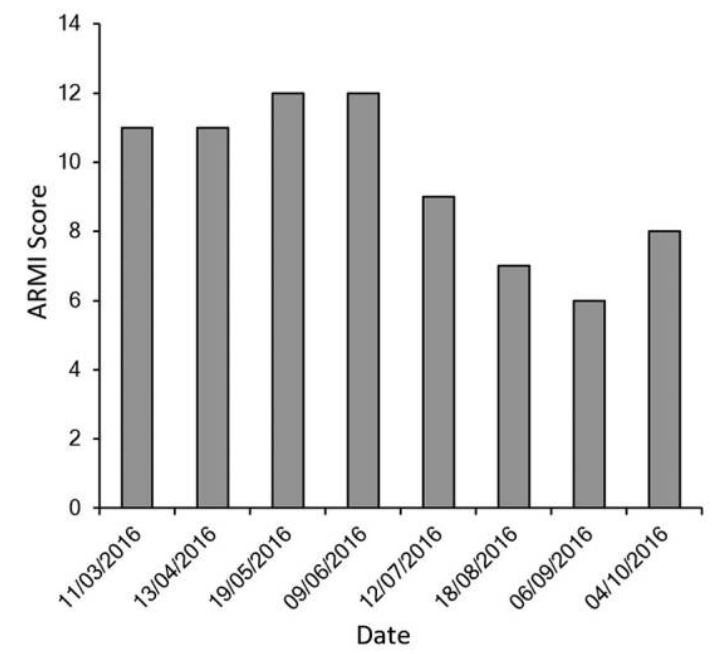

Figure 5. Anglers' Riverfly Monitoring Initiative (ARMI) scores from Broughton Beck Gatehouse, on the River Aire, North Yorkshire, UK in 2015 (A) and 2016 (B).

Volunteers from the Tyne Rivers Trust monitoring the River Derwent noticed ARMI scores dropped unexpectedly in February 2009. The long-term data available from the site suggested that there might be a water quality problem. There also had been a fish-kill in the river, although the score did not breach the trigger level at that site. An investigation by the EA established that a water treatment works had malfunctioned. Remediation work included improvements in the treatment works, habitat quality improvements, and fish restocking. ARMI scores returned to previous levels following the remediation.

The value the EA places on the reliability of volunteer ARMI monitoring is demonstrated in a $3^{\text {rd }}$ example from the Tyne Rivers Trust area. Following a period of high rainfall in mid-March 2010, the EA requested that ARMI volunteers conduct additional sampling on the Tipalt Burn because of concern that the high rainfall may have flushed heavy metals into the river from old mine workings. The ARMI scores for late March were lower than scores for earlier in the month, prior to the heavy rains, but the scores did not breach trigger levels set for the site. The ARMI scores returned to the expected levels by late April, which confirmed to the EA that they did not need to commit resources for remediation purposes.

River Bulbourne The River Bulbourne is a small chalk stream located in the Chiltern Hills, Hertfordshire, UK, and has a total length of $11.3 \mathrm{~km}$. The river receives water from the adjacent Grand Union Canal during periods of high rainfall. Between October 2015 and April 2016, ARMI scores at a sampling site immediately downstream of the Grand Union Canal inflow were low compared with ARMI scores observed in summer at both the same site and other sites along the river. The trigger level was breached in October 2015, when water was seen flowing into the river from the canal, and in December 2015 (Fig. 6). These trigger level breaches were associated with large reductions in the abundance of gammarids, Trichoptera, and Baetidae. The low ARMI scores were not caused by seasonal population fluctuations, as these taxa were still present in high abundance in other parts of the river at the same time of year. Instead the EA confirmed that the low scores were probably caused

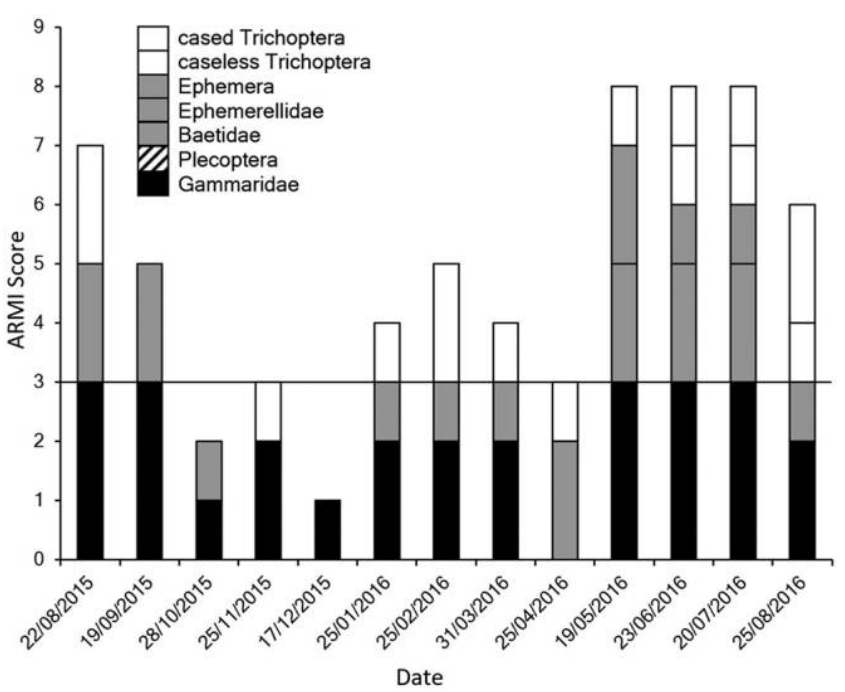

Figure 6. Anglers' Riverfly Monitoring Initiative (ARMI) scores in 2015-2016 from the River Bulbourne, Hemel Hempstead, Hertfordshire, UK, up- and downstream of the inlet from the Grand Union Canal. The horizontal line at ARMI score 3 represents the score that triggers action by the Environment Agency. 
by the flushing effect of a large volume of poor quality water entering the river from the canal.

\section{Sensitivity of ARMI to river restoration}

In addition to detecting the impacts of pollution incidents, ARMI can be used to show before-after responses to river restoration activities. An $\sim 1.6 \mathrm{~km}$ stretch of the river flows through the grazed water meadows of Boxmoor on the western edge of Hemel Hempstead, Hertfordshire. Regular monthly ARMI samples collected from 7 sites along the course of the river since August 2015 indicate that all but 2 sites maintained ARMI scores well above the trigger levels set by the EA. One of the sites, which was located immediately upstream of a small weir, produced ARMI scores that were consistently below the trigger level. The impounded water at this site was dominated by gammarids and did not support the high abundances of Trichoptera and Ephemeroptera that were recorded at the other sites monitored both further up- and downstream of the weir. In March 2016, as part of a river restoration program, the weir was removed to increase water velocity, and within 1 mo ARMI scores from the site upstream of the weir had increased and were similar to other sites on the river (Fig. 7). These high scores have been subsequently maintained at the site, which demonstrates the success of weir removal to improve the habitat quality of the river and also the ability of the ARMI system to detect this improvement.

\section{DISCUSSION}

ARMI provides a means for anglers and other volunteers to become more familiar with, and potentially enhance,

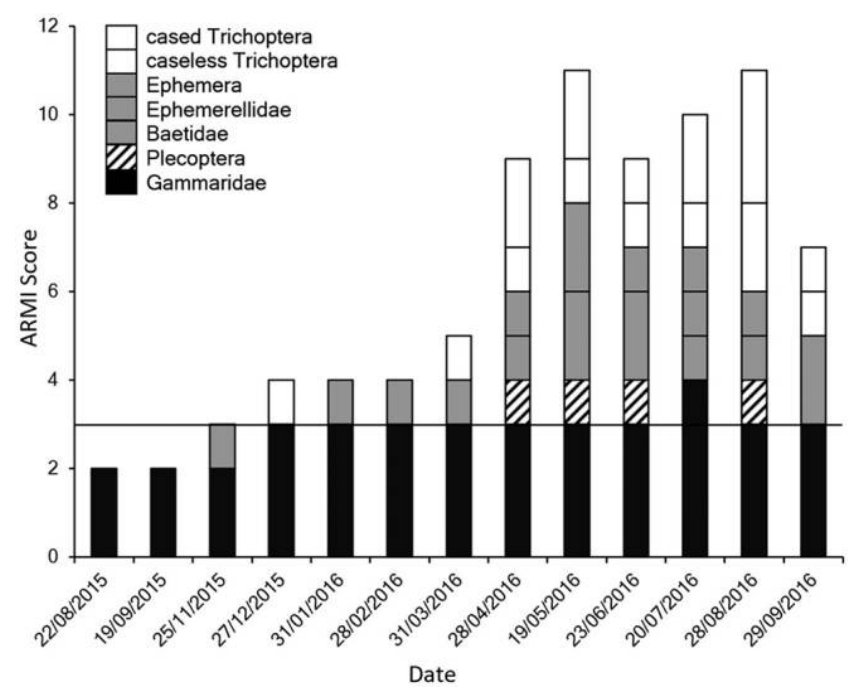

Figure 7. Anglers' Riverfly Monitoring Initiative (ARMI) scores in 2015-2016 for the River Bulbourne, Hemel Hempstead, Hertfordshire, UK, before and after weir removal. The horizontal line at ARMI score 3 represents the score that triggers action by the Environment Agency. their local river environment. Active ARMI volunteers that have a presence on their local rivers can become a focus for wider community engagement in, and appreciation of, the natural riparian environment. Further, those volunteers can become a nexus to build local community groups and increase community cohesion. Local community environmental action groups, which may include the ARMI among their portfolio of community projects, can provide a focal point to facilitate dialogue between local communities and the statutory regulatory authorities, including the EA and water companies. In turn, the local regulatory authorities can find working with a well-established and well-organized network of citizen scientists a useful, effective, and efficient way of delivering their statutory environmental obligations. In addition to providing basic ARMI monitoring, these groups of enthusiastic citizen scientists are willing and able to carry out additional specific monitoring work tailored to suit local requirements. The local regulatory authorities see the ARMI network as a resource that can be adapted to help them deliver their goals: a boon rather than a drain on their resources and staff time.

Additionally, participation in ARMI provides volunteers with the opportunity to become more familiar with scientific methods. ARMI volunteers become citizen scientists and use standardized monitoring protocols to produce robust data. Volunteers can then analyze their data both spatially and temporally alongside other such data collected from the same river catchment or even across the nation. This process provides volunteers with a new, transferable skill set and an improved understanding of the scientific method. This improved understanding can lead to enhanced scientific literacy-a vital skill in an increasingly science-based society.

ARMI provides an effective way of complementing routine statutory agency monitoring. Sites are visited more frequently by ARMI monitors than by the regulatory authorities, and on some rivers ARMI sites may also be established at higher spatial density than those monitored by the regulatory authority. Increased sampling frequency and density raises the chances of detecting pollution incidents that might otherwise go undetected, and also of pinpointing where the pollution source may be. In addition, the highly visible, regular, and frequent presence of ARMI monitors acts as a deterrent to would-be polluters. Long time-series of ARMI scores that do not breach trigger levels can also be useful in reassuring regulatory authorities that rivers are not deteriorating between their own less-frequent monitoring programs. These time series are also valuable in detecting chronic pollution and can be used as a cumulative evidence base that might later help to resolve pollution investigations. These datasets also provide important before and after information that can help determine recovery rates following major pollution incidents.

The analysis of BMWP scores from long-term biomonitoring data provided a test of the ARMI scoring sys- 
tem and demonstrated a close correlation between ARMI and BMWP scores. However, this analysis did not consider variation in results obtained from different individuals or groups within the ARMI scheme, or how groups or individuals could influence the scoring scheme. Unfortunately, no detailed analysis has been carried out to assess individual or group differences. However, there was prolonged pilot testing of the ARMI protocol prior to the national launch of the scheme, which involved many volunteer groups on many different river systems throughout England and Wales. These protocol tests indicated that the volunteers could, after $1 \mathrm{~d}$ of training, reliably perform the standardized sampling, identify the 8 taxa, and correctly analyze the data. Before results are uploaded to the database, it is quality assured by the river coordinator who will query unusual results. Additionally, before trigger breaches are notified to the regulatory authority a $2^{\text {nd }}$ sample is taken on the river, often by the river coordinator.

Volunteers provide a large return through in-kind benefits (estimated for the year 2015-2016 to be in excess of $£ 400,000$ ) (Environment Agency 2016). A local regulatory authority staff is needed to approve the suitability of ARMI sampling sites, set trigger levels for ARMI sites, and participate in ARMI training workshops. Oversight by the local regulatory authority minimizes the risk of false alarms and time wasted by the agency staff that investigates them, both because the trained volunteers are more likely to sample correctly and produce reliable ARMI scores and because the trigger levels are set to be sensitive to serious pollution incidents.

An important facet of any volunteer-based project is to ensure that volunteer motivation is maintained, thereby reducing the rate of volunteer turnover. ARMI offers various ways to keep volunteers motivated. ARMI volunteers know that the data they collect provide meaningful and useful information and that officers in the regulatory authority will act if a serious pollution incident is detected. Volunteers thus receive direct and prompt feedback following a trigger breach. The volunteers are engaged in a practical activity that helps to protect an environment they care about. Organizationally, ARMI is a bottom-up project, which results in volunteers having a sense of ownership over the project. Volunteers analyze the data they collect, rather than passing it on to a scientist who decides how the data are used. Therefore, by participating in ARMI, volunteers increase their skill set. Further, as the work is outdoors and involves some physical activity, it contributes positively to health and well-being and a sense of community between likeminded individuals. Together, these facets of ARMI serve to enhance volunteer motivation.

ARMI volunteer motivation is also promoted by working as part of a team. Pairs of volunteers monitor sites, and through the river coordinator, they keep in touch with other volunteers working on the same river. Additionally, at the regional hub and at national level they are able to stay connected through local and national meetings, newsletters, and the RP website and ARMI database. The national ARMI database provides an important way for volunteers to see how their data fit into the national picture and contribute to a broader national movement. The skills volunteers learn and the data they generate empower them by providing them with a means to engage in informed dialogue with officers in the regulatory authority.

Volunteers can also progress and increase their skills. At the entry level they learn to identify 8 invertebrate taxa, analyze data, and gain an understanding of the quality of the local river environment. Many volunteers soon want to develop their identification skills further and gain a more in-depth understanding of the river ecosystem. The Riverfly Plus add-ons provide a means for volunteers to do this. There are also opportunities for volunteers to become Riverfly tutors and train other volunteers, river coordinators and monitoring organizers on a particular river, or local Riverfly hub organizers.

ARMI provides citizens with a means to detect deteriorations in water quality, but most volunteers would like to see an improvement in river quality. Thus, many groups get involved in river restoration projects. It is essential to demonstrate ecosystem response to river restoration. Beforeafter-control-impact monitoring of restorations based on the ARMI method has been shown to be effective in demonstrating changes in riverfly diversity and abundance. Adoption of Riverfly Plus methods specifically designed for monitoring river restorations can be used in conjunction with basic ARMI monitoring methods to provide more nuanced evidence of river restoration success. Data on river restoration outcomes, gathered in a central database, will provide a useful resource when groups consider which restoration methods are most likely to produce desired results (Huddart et al. 2016).

ARMI has successfully attracted many volunteers across the UK and generated useful data on river water quality. In the coming years, RP will: 1) further demonstrate to the regulatory authorities that ARMI data are useful for their operations and that it is cost effective for them to continue supporting and engaging with the project, 2) continue to expand its network of volunteers with the objective of achieving uniform and high spatial coverage across the UK, 3) continue to develop Riverfly Plus and seek to increase the take up of these packages by ARMI volunteers, 4) interrogate the national database for long term trends in river quality and changes in riverfly abundance and distribution, and 5) further compare ARMI data with those routinely collected by the regulatory authorities.

\section{Conclusions}

The Anglers' Riverfly Monitoring Initiative is a successful citizen project that has engaged several thousand volunteers across the UK for $>10 \mathrm{y}$. ARMI volunteers have de- 
tected many pollution incidents that otherwise may not have been detected, which have prompted investigations by the regulatory authorities and remedial actions by the parties responsible for the pollution events. ARMI provides an effective means for community engagement with the river environment, and is an effective and efficient way for the regulatory authorities to: 1) engage with a network of stakeholders who may undertake further types of river monitoring, 2) obtain river water quality data that complements their own data collection, 3) monitor alerts and pinpoint pollution sources, 4) gather information on the spatial extent and timing of pollution incidents that is critical for their own investigations, 5) receive information about pollution incidents, and 6) deter would-be polluters through increased surveillance. As a whole, the ARMI demonstrates the potential utility and cost effectiveness of citizen scientists working closely and cooperatively with the regulatory authorities. In particular, the frequent, regular, and standardized monitoring allows ARMI to provide robust and reliable long-term data that complement and augment the routine monitoring of the regulatory authorities.

\section{ACKNOWLEDGEMENTS}

Author contributions. SJB conceived and designed the paper and wrote the bulk of the text. BF made a substantial contribution to writing the text and compiled case study details. JD-B and SAC analyzed the ARMI and BMWP data and contributed to later drafts of the text. JD-B redrew the figures.

We thank Richard Chadd and Chris Extence (Environment Agency) for information on their slow flow monitoring protocol based on 26 taxa. We thank Rob Gray, Joe Pecorelli, and Richard Haine for providing us with information on the River Crane project. We thank Dr Murray Thompson for information on the River Kennet insecticide spillage. We thank Joanna Hood (EA) and Ian Mulley for information on the case studies from North Yorkshire. We thank Ceri Gibson and the Tyne Rivers Trust for providing case study information. We thank Allen Beechey for information on the Chilterns case studies. We thank 2 anonymous reviewers for the helpful and constructive comments that helped improve the quality of the paper.

\section{LITERATURE CITED}

Bonney, R., C. B. Cooper, J. Dickinson, S. Kelling, T. Phillips, K. V. Rosenberg, and J. Shirk. 2009. Citizen science: a developing tool for expanding science knowledge and scientific literacy. BioScience 59:977-984.

Di Fiore, D., and B. R. N. Fitch. 2016. The riverfly monitoring initiative: structured community data gathering informing statutory responses. Environmental Scientist 25:36-41.

Environment Agency. 2016. Annual fisheries report 2015-2016. Environment Agency, Bristol, UK. (Available from: nflg.co.uk /files/LIT_10620.pdf)

Environment Agency and English Nature. 2004. The state of England's chalk rivers: a report by the UK Biodiversity Action Plan Steering Group for Chalk Rivers. Environment Agency, Bristol, UK.
Gowan, C., M., R. R. Knisley, and L. Grimme. 2007. Stream monitoring methods suitable for citizen volunteers working in the Coastal Plain and Lower Piedmont regions of Virginia. American Entomologist 53:48-57.

Hawkes, H. A. 1997. Origin and development of the Biological Monitoring Working Party score system. Water Research 32:964-968.

Huddart, J. E. A., M. S. A. Thompson, G. Woodward, and S. J. Brooks. 2016. Citizen science: from detecting pollution to evaluating ecological restoration. WIREs Water 3:287-300.

Latimore, J. A., and P. J. Steen. 2014. Integrating freshwater science and local management through volunteer monitoring partnerships: the Michigan Clean Water Corps. Freshwater Science 33:686-692.

Moffett, E. R., and M. W. Neale. 2015. Volunteer and professional macroinvertebrate monitoring provide concordant assessments of stream health. New Zealand Journal of Marine and Freshwater Research 49:366-375.

Overdevest, C., C. H. Orr, and K. Stepenuck. 2004. Volunteer stream monitoring and local participation in natural resource issues. Human Ecology Review 11:177-185.

Paisley, M. F., D. J. Trigg, and W. J. Walley. 2007. Revision and testing of BMWP scores. Final report SNIFFER Project WFD72a. SNIFFER, Edinburgh, UK. (Available from: http://eprints.bourne mouth.ac.uk/16550/)

Reynoldson, T., L. Hampel, and J. Martin. 1986. Biomonitoring networks operated by schoolchildren. Environmental Pollution Series A: Ecological and Biological 41:363-380.

Roy, H. E., M. J. O. Pocock, C. D. Preston, D. B. Roy, J. Savage, J. C. Tweddle, and L. D. Robinson. 2012. Understanding citizen science and environmental monitoring. Final Report on behalf of UK-EOF. NERC Centre for Ecology and Hydrology, Wallingford and Hydrology and Natural History Museum, London, UK.

Savan, B., A. J. Morgan, and C. Gore. 2003. Volunteer environmental monitoring and the role of the universities: the case of Citizens' Environment Watch. Environmental Management 31:561-568.

Storey, R. G., A. Wright-Stow, E. Kin, R. Davies-Colley, and R. Stott. 2016. Volunteer stream monitoring: do the data quality and monitoring experience support increased community involvement in freshwater decision making? Ecology and Society 21:32.

Thompson, M. S. A., C. Bankier, T. Bell, A. J . Dumbrell, C. Gray, M. E. Ledger, K. Lehmann, B. A. McKew, C. D. Sayer, F. Shelley, M. Trimmer, S. L. Warren, and G. Woodward. 2015. Gene-to-ecosystem impacts of a catastrophic pesticide spill: testing a multilevel bioassessment approach in a river ecosystem. Freshwater Biology 61:2037-2050.

Walley, W. J., and H. A. Hawkes. 1996. A computer-based reappraisal of the Biological Monitoring Working Party scores using data from the 1990 river quality survey of England and Wales. Water Research 30:2086-2094.

Walley, W. J., and H. A. Hawkes. 1997. A computer-based reappraisal of the Biological Monitoring Working Party score system incorporating abundance rating, site type and indicator value. Water Research 31:201-210.

WWF-UK. 2014. The state of England's chalk streams. (Available from: assets.wwf.org.uk/downloads/wwf_chalkstreamreport _final_lr.pdf) 